\title{
(2) OPEN ACCESS \\ Abdominal cyst of unclear aetiology: gastrointestinal stromal tumour or reactivation of abdominal tuberculosis
}

\author{
Damini Saxena 이, ${ }^{1}$ Robert A Duncan, ${ }^{2}$ Robert R Faust, ${ }^{1}$ Anthony Campagna ${ }^{3}$
}

${ }^{1}$ General Internal Medicine, Lahey Hospital and Medical Center, Burlington, Massachusetts, USA ${ }^{2}$ Infectious Diseases, Lahey Hospital and Medical Center, Burlington, Massachusetts, USA ${ }^{3}$ Pulmonary and Critical Care Medicine, Lahey Hospital and Medical Center, Burlington, Massachusetts, USA

Correspondence to Dr Damini Saxena; dsax00@gmail.com

Accepted 5 December 2021
D) Check for updates

(c) BMJ Publishing Group Limited 2022. Re-use permitted under CC BY-NC. No commercial re-use. See rights and permissions. Published by BMJ.

To cite: Saxena $D$ Duncan RA, Faust RR, et al. BMJ Case Rep 2022:15:e245767. doi:10.1136/bcr-2021 245767

\section{SUMMARY}

Differential diagnosis of a new abdominal mass is broad and includes infection, malignancy and other inflammatory processes. Definitive diagnosis may be challenging without invasive biopsy, as history, physical exam and imaging may be non-specific. A 69-year-old man with a history of abdominal tuberculosis presented with a new painful abdominal cyst consistent with reactivation of tuberculosis versus new malignancy. Investigations revealed 4+ acid-fast bacilli from the aspirate suggestive of tuberculosis, but no improvement was noted on antituberculous therapy. Core needle biopsy noted c-KIT-positive spindle cells, diagnostic for a gastrointestinal stromal tumour, while cultures grew nontuberculous mycobacteria.

\section{BACKGROUND}

Approximately one-fourth of the world's population is infected with tuberculosis (TB). ${ }^{1}$ Extrapulmonary TB is infrequent, accounting for about $15 \%$ of all new TB cases worldwide in $2016 .^{2}$ Abdominal TB is even rarer, totalling only $1 \%-3 \%$ of all TB cases globally. ${ }^{3}$ It is notoriously difficult to diagnose abdominal TB due to its non-specific signs and symptoms, as it typically presents with abdominal pain and other vague constitutional symptoms. ${ }^{4}$ Further, it is often challenging to isolate acid-fast bacilli (AFB) from patient specimens. ${ }^{4}$ Abdominal TB is best imaged by CT imaging, which typically reveals asymmetric wall thickening of the gastrointestinal tract, sometimes with necrotic lymph nodes. ${ }^{5}$ Diagnosis needs to be made promptly to allow for early initiation of antituberculous treatment to prevent the morbidity and mortality of untreated TB. Definitive diagnosis of abdominal TB relies on needle biopsy with histopathology and mycobacterial cultures from the specimen. ${ }^{4}$

Given its challenges in diagnosis, abdominal TB must be distinguished from a broad differential diagnosis of abdominal pathologies, including Crohn's disease, malignancies, other acute infections and intestinal sarcoidosis. ${ }^{3}$ In fact, gastrointestinal stromal tumours (GISTs) have remarkably similar diagnostic challenges to abdominal TB. Patients with GISTs often present with similar abdominal symptoms and/or constitutional complaints. ${ }^{5}$ Larger GISTs may be visualised on CT as heterogeneous masses in the luminal wall, often accompanied by necrosis. ${ }^{5}$ Due to insufficient sampling, diagnosis of GISTs on aspirated samples alone may be inadequate, similar to abdominal TB. ${ }^{5}$ Abdominal TB misdiagnosed as GIST has been reported previously. ${ }^{67}$ An early diagnosis of GIST may be lifesaving as standard therapy with surgical resection with or without immunotherapy has 5 -year survival rates of over $90 \%$ in localised disease but only $55 \%$ in the setting of distant metastasis. ${ }^{8}$

\section{CASE PRESENTATION}

A 69-year-old man from Asia presented with left upper quadrant (LUQ) abdominal pain. His medical history was most notable for a diagnosis of ileocecal TB in 1993 based on appearance on CT scan. At that time, he was treated with 10 months of isoniazid, rifampin, pyrazinamide (PZA) and ethambutol (RIPE), with reported resolution of imaging findings. While visiting family in the United States in 2019, he developed isolated LUQ abdominal pain without other signs or symptoms. On examination, a mass was palpated lateral to the stomach. Blood work revealed a mild normocytic anaemia but otherwise normal complete blood count and electrolytes. C reactive protein was elevated to $37 \mathrm{mg} / \mathrm{L}$ on presentation. CT scan revealed a complex cyst measuring $13 \times 11 \times 10 \mathrm{~cm}$ abutting the stomach and spleen, concerning for a pyogenic abscess (figure 1). Aspiration of the lesion revealed sanguinous fluid and $6 \times 10^{9}$ white blood cells per litre with neutrophilic predominance. Gram stain was unrevealing, while AFB and anaerobic cultures yielded no growth. Fungal, aerobic and anaerobic blood cultures remained unremarkable. Cytology of the fluid showed no malignant cells. At the time of the needle aspiration, a drain was placed for decompression of the abscess. Because of reaccumulation of fluid, drainage was performed again 2 weeks later.

\section{INVESTIGATIONS}

Repeat drainage revealed $4+\mathrm{AFB}$ on acid fast smear. AFB culture could not be grown, though, on solid or liquid media. QuantiFERON TB gold was positive. HIV-1 and 2, Echinococcus and Entamoeba histolytica serologies were negative. Based on his reported history of ileocecal TB and preliminary microbiological findings, the patient was diagnosed with reactivation of abdominal TB and started on RIPE therapy with supplementary pyridoxine.

Ten days later, his treatment was complicated by elevation of transaminases and all RIPE therapy was discontinued. RIPE was then sequentially re-introduced and the offending agent was found to be PZA. Moxifloxacin was substituted for PZA, and 


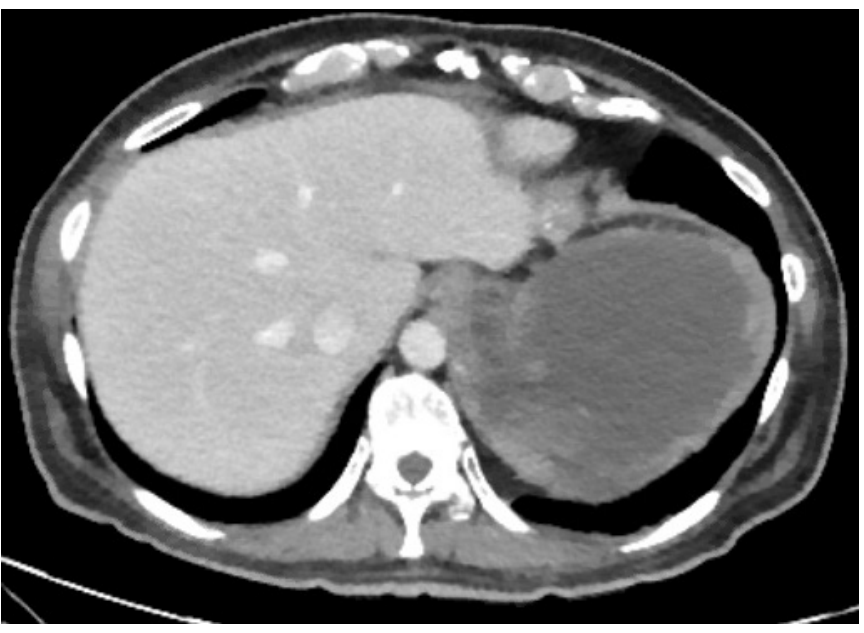

Figure 1 Abdominal CT scan showed a large complex cyst abutting the stomach and spleen

the transaminitis resolved. Mycobacterial cultures from the aspirates remained challenging to grow, complicated by overgrowth of normal bowel flora.

One month after reintroduction of antituberculous therapy, the patient reported increased abdominal drainage, associated with worsening anorexia, hypotension and hypovolaemic hyponatraemia. This prompted a repeat CT of the abdomen, showing that the mass was enlarging with increasing areas of wall thickening and soft tissue nodularity, suggesting an increasingly organised collection (figure 2).

A core needle tissue biopsy was obtained through interventional radiology. Histopathology illustrated soft tissue cores of spindle cell proliferation that stained positively for CD117 (c-KIT) and CD34, consistent with GIST (figure 3). Repeat cyst cultures grew Pseudomonas putida and Mycobacterium chelonae, which was the likely aetiology of the positive AFB smears.

\section{DIFFERENTIAL DIAGNOSIS}

Differential diagnosis for an abdominal mass is broad and includes infection, malignancy and other inflammatory processes. Given elevated C reactive protein level, an inflammatory process was high on the differential diagnosis. The mass on initial CT scan was concerning for a pyogenic abscess, thus an infectious process was considered to be most likely. Since the patient had

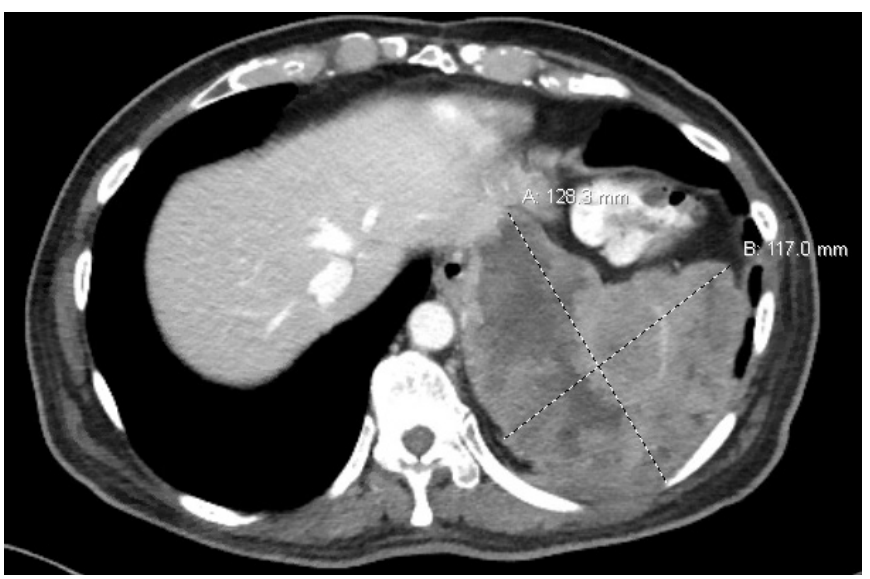

Figure 2 Subsequent CT scan noted enlarging mass with a necrotic centre and wall thickening

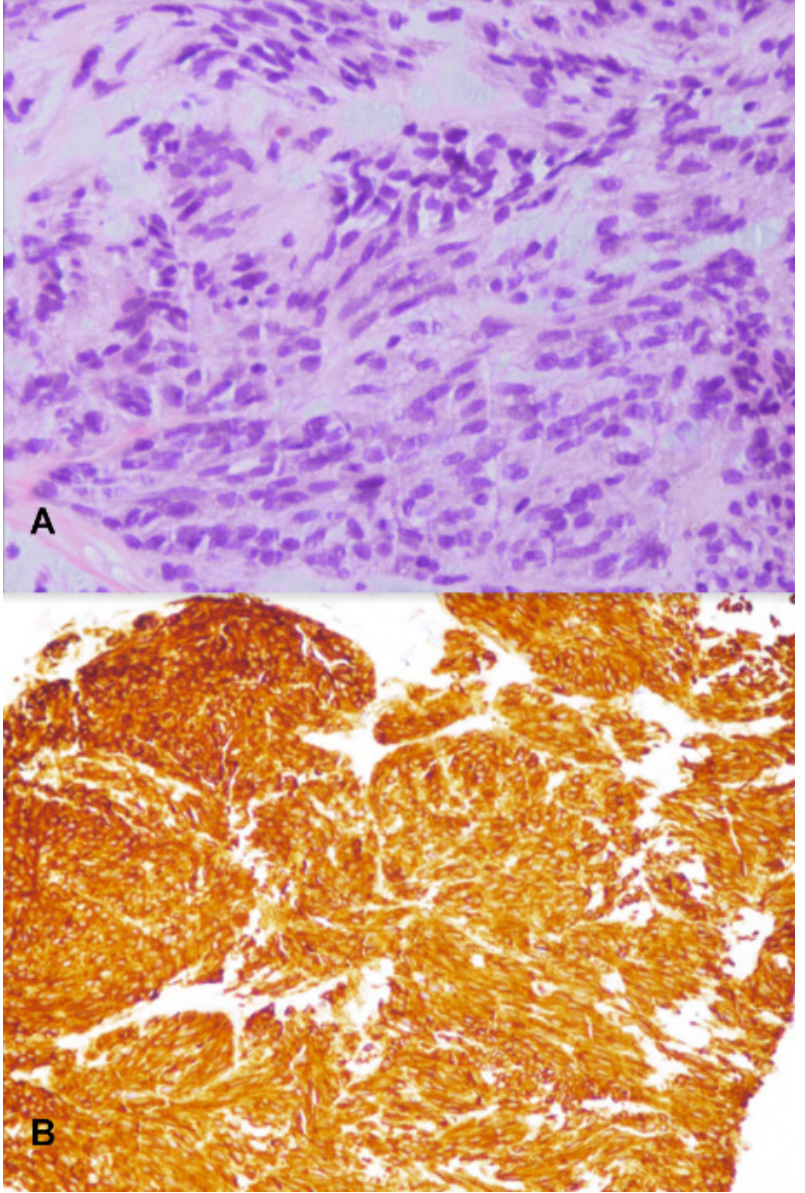

Figure 3 Biopsy showed spindle cell proliferation on (A) hematoxylin and eosin stain, magnification 20x, and (B) CD117+immunostaining, magnification $10 x$.

a history of abdominal $\mathrm{TB}$, reactivation of $\mathrm{TB}$ was of strong concern, prompting AFB smear and cultures to be obtained. The patient's risk factors for abdominal TB combined with $4+\mathrm{AFB}$ on acid fast smear were strongly suggestive for a diagnosis of TB infection. Remarkably, the patient's symptoms did not improve with antituberculous therapy and AFB cultures did not reveal Mycobacterium tuberculosis, prompting biopsy of the mass. Histopathology of the core needle biopsy strongly corroborated a diagnosis of GIST. However, a diagnosis of coinfection with $M$. tuberculosis was still under consideration due to the presence of AFB on smear. Repeat cultures revealed $M$. chelonae, explaining the presence of AFB on smear and a diagnosis of M. tuberculosis was eliminated from the differential.

\section{TREATMENT}

On initial aspiration of the mass, no definitive diagnosis was found. The patient was managed supportively only, with continuous drainage of the mass and outpatient follow-up. When $4+\mathrm{AFB}$ was detected on acid fast smear after repeat aspiration, the patient was treated with RIPE therapy for presumed diagnosis of M. tuberculosis. Moxifloxacin was substituted in for PZA subsequently due to the development of transaminitis from PZA use. Once the diagnosis of $M$. chelonae was made on biopsy, antituberculous treatment was discontinued and oral clarithromycin was started instead. P. putida was assessed to be a commensal organism that was unlikely to be causing infection. 
Once the diagnosis of GIST was made on biopsy, staging and referral to oncology and general surgery were recommended. The patient wished to proceed with definitive care in his home country, thus staging and specialty referrals were not pursued in the United States.

\section{OUTCOME AND FOLLOW-UP}

The patient wished to proceed with further care at home. Within a couple of days of diagnosis of GIST, he travelled to his home country in stable condition. He was evaluated by his primary care provider and general surgery in his home country. $\mathrm{He}$ started treatment for GIST at that time. One year later, however, he developed oncological complications that resulted in death.

\section{DISCUSSION}

This case highlights several important points. First, the diagnosis of reactivation of TB hinges on high clinical suspicion. This patient was at increased risk for TB given he had presented from a highly endemic area with prior presumptive diagnosis and resolution. His current laboratory workup showing positive QuantiFERON gold testing was consistent with a diagnosis of $\mathrm{TB}$, though this result cannot be further extrapolated to determine active versus latent TB. Positive AFB smears are more consistent with an active infection. Although AFB smears have a specificity approaching $97 \%$ for mycobacteria,${ }^{910}$ they cannot distinguish between tuberculous and non-tuberculous mycobacteria. ${ }^{10}$ Thus, cultures growing $M$. tuberculosis become necessary in the definitive diagnosis of active TB.

This warrants explanation of the second teaching point of this case. It is notoriously difficult to grow mycobacteria on cultures in the setting of abdominal TB. The sensitivity of detecting pulmonary TB on a single sputum culture is around $79 \% .{ }^{11} \mathrm{In}$ contrast, only $20 \%$ of cultures will successfully grow mycobacteria from abdominal specimens. ${ }^{12}$ Abdominal TB becomes even more challenging to diagnose because up to $15 \%$ of patients will not have associated pulmonary disease, and sampling the sputum cannot assist in diagnosis. ${ }^{13}$

Multiple new techniques have been developed to improve definitive diagnosis of abdominal TB. These include mycobacteria growth indicator tube (MGIT), PCR, Xpert MTB/RIF assay and TB-loop mediated isothermal amplification (TBLAMP). ${ }^{14}$ However, clinical applications of these techniques are not well established. MGIT is a liquid-based media that uses fluorochromes to detect growth and drug susceptibility and has been faster at yielding growth with less cross-contamination. Despite this advancement, the positive culture yield has only been around $51 \%-53 \%$ on MGIT media. ${ }^{14}$ Addition of certain antibiotics, such as p-nitrobenzoic acid (PNB), may help distinguish TB from non-tuberculous mycobacteria on MGIT, as PNB selectively inhibits $\mathrm{TB}$ growth while allowing non-tuberculous mycobacteria to proliferate. ${ }^{15}$ Selective testing of various mycobacterial species on MGIT, though, is not a routine practice. PCR was initially believed to be a promising approach with high specificity, but is dependent on sample quantity and quality. The Xpert MTB/RIF assay uses nucleic acid amplification techniques to rapidly detect $\mathrm{TB}$ and potential resistance to rifampin. It has a reported sensitivity and specificity of $88 \%$ and $91 \%$ for extrapulmonary TB, respectively, but is not a replacement for mycobacterial cultures or growth-based susceptibility tests required for adequate treatment of TB. ${ }^{14} 16$ TB-LAMP is a cutting edge technique that appears useful for earlier diagnosis of TB but has not yet been clinically validated for extrapulmonary TB.
Abdominal TB may look remarkably similar to GIST but has a drastically different pathophysiology. Abdominal TB is most often found in the ileocecal region after AFB in the respiratory system are coughed up and ingested in the gastrointestinal tract or from AFB being consumed in infected milk..$^{13} \mathrm{M}$. tuberculosis can spread hematogenously and form thickened submucosal granulomas that can ulcerate. ${ }^{13}$ These may present in remarkably similar fashion to GISTs with symptoms including abdominal pain, gastrointestinal bleeding and ulceration. On imaging, CT for both TB and GIST may show thickened luminal walls. ${ }^{13} 17$ Similar to our patient, M. chelonae may be seen as a disseminated disease in patients with cancer, such as GIST, which further contributes to diagnostic hesitancy. ${ }^{18}$ The stark contrast between them occurs at the microscopic level. Most GISTs are made up of spindle cells staining palely eosinophilic that contain vacuoles adjacent to round nuclei. ${ }^{17}$ They tend to be sclerotic, arranged in whorls or fascicles, and collagenous. ${ }^{17}$ In contrast, abdominal TB has characteristic granulomas with or without necrosis on histological examination. ${ }^{19}$ Caseation is more common in lymph nodes than in the gastrointestinal mucosa, and AFB may also be seen in histological specimens. ${ }^{19}$ This suggests that in most patients, histological analysis is the most definitive way of distinguishing between GIST and abdominal TB.

This case exemplifies that the diagnosis of a new painful abdominal mass, in a patient with previous TB, requires meticulous history taking, diagnostic work up and often invasive histological analysis. Distinguishing abdominal TB from other causes of painful abdominal mass is challenging but necessary, as early initiation of appropriate treatment can be life saving and reduce morbidity. Analysis of newer non-invasive techniques to isolate M. tuberculosis appear promising but need to be further validated prior to clinical use.

\section{Learning points}

- Definitive diagnosis of abdominal tuberculosis from gastrointestinal stromal tumour may be difficult, as both present with similar clinical features and imaging findings.

- In histopathology, gastrointestinal stromal tumours will show spindle cells that may be c-KIT positive, whereas abdominal tuberculosis will present with granulomas with or without caseation.

- Despite many emerging techniques, culturing mycobacteria from abdominal samples remains a significant challenge.

Twitter Damini Saxena @DSaxena_MD

Acknowledgements The authors would like to thank Dr Namrata Anand in the Department of Anatomic Pathology at Beth Israel Lahey Health for her guidance on the histological findings in this case.

Contributors DS was the resident physician caring for the patient and the primary writer of the manuscript. RAD was the infectious disease specialist caring for the patient and contributed to the writing of the manuscript. RRF was the patient's primary care physician and contributed to the writing of the manuscript. AC was the patient's primary pulmonologist and contributed to the writing of the manuscript. All authors were in agreement with the contents of the manuscript prior to submission.

Funding The authors have not declared a specific grant for this research from any funding agency in the public, commercial or not-for-profit sectors.

Competing interests None declared.

Patient consent for publication Consent obtained from next of kin. Provenance and peer review Not commissioned; externally peer reviewed.

Open access This is an open access article distributed in accordance with the Creative Commons Attribution Non Commercial (CC BY-NC 4.0) license, which permits others to distribute, remix, adapt, build upon this work non-commercially, 
and license their derivative works on different terms, provided the original work is properly cited and the use is non-commercial. See: http://creativecommons.org/ licenses/by-nc/4.0/.

Case reports provide a valuable learning resource for the scientific community and can indicate areas of interest for future research. They should not be used in isolation to guide treatment choices or public health policy.

\section{ORCID iD}

Damini Saxena http://orcid.org/0000-0002-1263-9990

\section{REFERENCES}

1 Self-study modules on tuberculosis: epidemiology of tuberculosis. CDC.gov 2019. Available: https://www.cdc.gov/tb/education/ssmodules/pdfs/module2.pdf [Accessed 23 Jul 2021].

2 Ayed HB, Koubaa M, Marrakchi C. Extrapulmonary tuberculosis: update on the epidemiology, risk factors, and prevention strategies. Int J Trop Med 2018;1.

3 Chakinala RC, Khatri AM. Gastrointestinal Tuberculosis. StatPearls Online [eLetter] 12 May 2021. Available: https://www.ncbi.n/m.nih.gov/books/NBK556115/

4 Rai S, Thomas WM. Diagnosis of abdominal tuberculosis: the importance of laparoscopy. J R Soc Med 2003;96:586-8.

5 Hong X, Choi H, Loyer EM, et al. Gastrointestinal stromal tumor: role of CT in diagnosis and in response evaluation and surveillance after treatment with imatinib. Radiographics 2006;26:481-95.

6 Kim YS, Moon JS, Lee JW, et al. Solitary intra-abdominal tuberculous lymphadenopathy mimicking duodenal GIST. Korean J Intern Med 2005;20:72-5.

7 Gupta V, Goel MM, Noushif M, et al. Primary gastric tuberculosis mimicking gastrointestinal stromal tumor. Am J Gastroenterol 2012;107:1269-70.
8 Gastrointestinal stromal tumor - GIST: Statistics, 2021. Cancer.net Online [eLetter] 23 July 2021. Available: https://www.cancer.net/cancer-types/gastrointestinal-stromaltumor- gist/statistics

9 Afsar I, Gunes M, Er H, et al. Comparison of culture, microscopic smear and molecular methods in diagnosis of tuberculosis. Rev Esp Quimioter 2018;31:435-8.

10 Bahammam A, Choudhri S, Long R. The validity of acid-fast smears of gastric aspirates as an indicator of pulmonary tuberculosis. Int J Tuberc Lung Dis 1999;3:62-7.

11 Parker RA. Implications of tuberculosis sputum culture test sensitivity on accuracy of other diagnostic modalities. Am J Respir Crit Care Med 2018;199.

12 Debi U, Ravisankar V, Prasad KK, et al. Abdominal tuberculosis of the gastrointestinal tract: revisited. World J Gastroenterol 2014;20:14831-40.

13 Rodriguez-Takeuchi SY, Renjifo ME, Medina FJ. Extrapulmonary tuberculosis: pathophysiology and imaging findings. Radiographics 2019;39:2023-37.

14 Mehta V, Desai D, Abraham P, et al. Making a positive diagnosis of intestinal tuberculosis with the aid of new biologic and histologic features: how far have we reached? Inflamm Intest Dis 2019;3:155-60.

15 Sharma B, Pal N, Malhotra B, et al. Evaluation of a rapid differentiation test for Mycobacterium tuberculosis from other mycobacteria by selective inhibition with p-nitrobenzoic acid using MGIT 960. J Lab Physicians 2010;2:089-92.

16 A new tool to diagnose tuberculosis: the Xpert MTB/RIF assay. Atlanta (GA): centers for disease control and prevention, 2016. Available: https://www.cdc.gov/tb/ publications/factsheets/pdf/xpertmtb-rifassayfactsheet_final.pdf [Accessed 23 Jul 2021].

17 Foo WC, Liegl-Atzwanger B, Lazar AJ. Pathology of gastrointestinal stromal tumors. Clin Med Insights Pathol 2012;5:CPath.S9689-33.

18 Akram SM, Ratish B, Saleh D. Mycobacterium chelonae. StatPear/s Online [eLetter] 23 July 2021. Available: https://www.ncbi.nlm.nih.gov/books/NBK430806/

19 Dasgupta A, Singh N, Bhatia A. Abdominal tuberculosis: a histopathological study with special reference to intestinal perforation and mesenteric vasculopathy. J Lab Physicians 2009:1:056-61.

Copyright 2021 BMJ Publishing Group. All rights reserved. For permission to reuse any of this content visit https://www.bmj.com/company/products-services/rights-and-licensing/permissions/ BMJ Case Report Fellows may re-use this article for personal use and teaching without any further permission.

Become a Fellow of BMJ Case Reports today and you can:

- Submit as many cases as you like

- Enjoy fast sympathetic peer review and rapid publication of accepted articles

- Access all the published articles

- Re-use any of the published material for personal use and teaching without further permission

Customer Service

If you have any further queries about your subscription, please contact our customer services team on +44 (0) 2071111105 or via email at support@bmj.com. Visit casereports.bmj.com for more articles like this and to become a Fellow 